\title{
Magnetic and Structural Studies of the Quasi-Two-Dimensional Spin-Gap System $(\mathrm{CuCl}) \mathrm{LaNb}_{2} \mathrm{O}_{7}$
}

\author{
Makoto YoshidA $^{1 *}$, Nobuyuki Ogata ${ }^{1}$, Masashi Takigawa ${ }^{1 \dagger}$, Jun-ichi Yamaura ${ }^{1}$, Masaki Ichinara ${ }^{1}$, Taro \\ Kitano $^{2}$, Hiroshi KageYama ${ }^{2}$, Yoshitami AJiro ${ }^{2}$, and Kazuyoshi Yoshimura ${ }^{2}$ \\ ${ }^{1}$ Institute for Solid State Physics, University of Tokyo, Kashiwa, Chiba 277-8581 \\ ${ }^{2}$ Department of Chemistry, Graduate School of Science, Kyoto University, Kyoto 606-8502
}

\begin{abstract}
We report magnetization, nuclear magnetic resonance (NMR), nuclear quadrupole resonance (NQR), and transmission electron microscopy (TEM) studies on the quasi-two-dimensional spin-gap system $(\mathrm{CuCl}) \mathrm{LaNb}_{2} \mathrm{O}_{7}$, a possible candidate for the $J_{1}-J_{2}$ model on a square lattice. A sharp single $\mathrm{NQR}$ line is observed at the $\mathrm{Cu}$ and $\mathrm{Cl}$ sites, indicating that both $\mathrm{Cu}$ and $\mathrm{Cl}$ atoms occupy a unique site. However, the electric field gradient tensors at the $\mathrm{Cu}, \mathrm{Cl}$, and La sites do not have axial symmetry. This is incompatible with the reported crystal structure. Thus the $J_{1}-J_{2}$ model has to be modified. We propose alternative two-dimensional dimer models based on the NMR, NQR, and TEM results. The value of the hyperfine coupling constant at the $\mathrm{Cu}$ sites indicates that the spin density is mainly on the $d\left(3 z^{2}-r^{2}\right)$ orbital $(z \| c)$. At 1.5 $\mathrm{K}, \mathrm{Cu}$ - and $\mathrm{Nb}-\mathrm{NMR}$ signals disappear above the critical field $B_{c 1} \simeq 10.3 \mathrm{~T}$ determined from the onset of the magnetization, indicating a field-induced magnetic phase transition at $B_{c 1}$.
\end{abstract}

KEYWORDS: NMR, NQR, TEM, $(\mathrm{CuCl}) \mathrm{LaNb}_{2} \mathrm{O}_{7}$, spin-gap, quantum spin system

\section{Introduction}

Low-dimensional quantum spin systems with frustrating interactions have recently been studied intensively both from experimental and theoretical aspects. The $S=$ $1 / 2$ antiferromagnet on a square lattice with the nearestneighbor and the next-nearest-neighbor interactions (the $J_{1}-J_{2}$ model) is a well known example. When the nearest neighbor interaction $J_{1}$ is antiferromagnetic $\left(J_{1}>0\right)$, the ground state has a Néel order with the wave vector $(\pi, \pi)$ for $J_{2} / J_{1}<0.38$ while a collinear order with the wave vector $(\pi, 0)$ occurs for $J_{2} / J_{1}>0.52 .^{1-3)}$ A spin liquid phase is proposed between these phases near $J_{2} / J_{1}$ $\simeq 0.5 .^{1-3)}$ A spin liquid phase is also proposed for ferromagnetic $J_{1}\left(J_{1}<0\right)$ between the collinear phase for $J_{2} / J_{1}<-0.51$ and the ferromagnetic phase for larger $J_{2} / J_{1} \cdot{ }^{3)}$ The series of compounds $(\mathrm{Cu} X) \mathrm{LaNb}_{2} \mathrm{O}_{7}(X=$ $\mathrm{Cl}, \mathrm{Br})$ is a candidate for the $J_{1}-J_{2}$ model with mixed ferro- and antiferromagnetic interactions. ${ }^{4-6)}$ The structure is reported to be tetragonal (space group $P 4 / \mathrm{mmm}$ ) with the $\mathrm{Cu} X$ square lattice ${ }^{7,8)}$ as shown in Fig. 1. In fact, $(\mathrm{CuCl}) \mathrm{LaNb}_{2} \mathrm{O}_{7}$ has a singlet ground state with a spin-gap ${ }^{4,5)}$ while $(\mathrm{CuBr}) \mathrm{LaNb}_{2} \mathrm{O}_{7}$ shows a collinear antiferromagnetic order. $\left.{ }^{6}\right)$

In $(\mathrm{CuCl}) \mathrm{LaNb}_{2} \mathrm{O}_{7}$, the spin-gap is estimated to be 27 $\mathrm{K}$ by fitting the susceptibility data to the isolated dimer model. ${ }^{4)}$ The neutron inelastic scattering results also exhibit magnetic excitations with the gap of $2.3 \mathrm{meV}$, although the resolution is limited. $\left.{ }^{4}\right)$ On the other hand, the high-field magnetization measurements revealed a sudden increase at the critical field $B_{c 1}=10.3 \mathrm{~T}$. ${ }^{5}$ ) The Zeeman energy at $B_{c 1}, g \mu_{\mathrm{B}} B_{c 1} / k_{\mathrm{B}}=15 \mathrm{~K}$, is apparently not sufficient to close the spin-gap at zero field. In addition, the magnetization saturates at $B_{c 2}=30.1 \mathrm{~T} .{ }^{5)}$ The large interval between $B_{c 1}$ and $B_{c 2}$ indicates a large

*E-mail address: yopida@issp.u-tokyo.ac.jp

†E-mail address: masashi@issp.u-tokyo.ac.jp

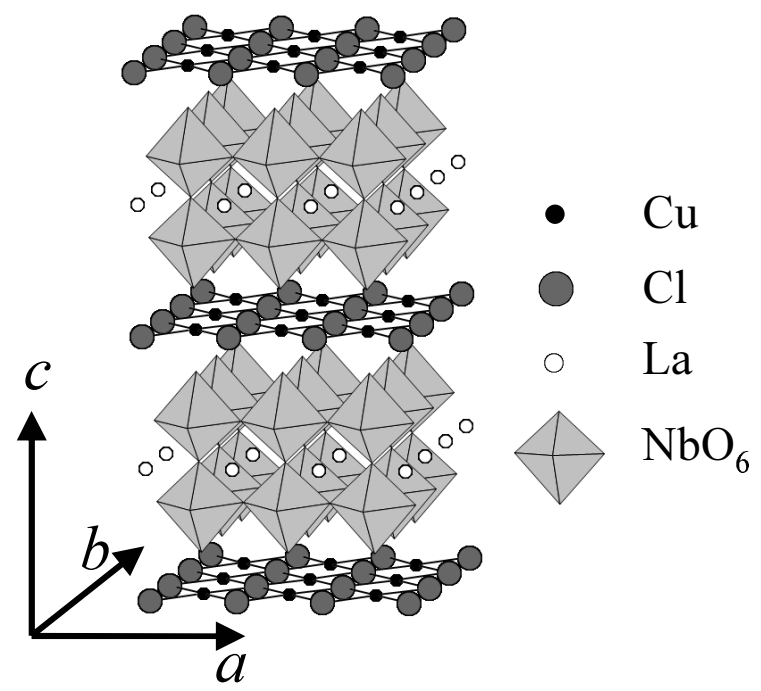

Fig. 1. Schematic drawing of the crystal structure of $\left.(\mathrm{CuCl}) \mathrm{LaNb}_{2} \mathrm{O}_{7} \cdot{ }^{7}\right)$

width of the triplet dispersion. This is inconsistent with the neutron inelastic scattering data indicating that the triplet mode is almost dispersionless. ${ }^{4}$ )

There are also open issues about the crystal structure. The neutron diffraction study indicates that the $\mathrm{Cl}$ position deviates from the ideal $(0,0,1 / 2)$ position with the $\mathrm{C}_{4}$-symmetry in the tetragonal space group $P 4 / \mathrm{mmm}^{8}{ }^{8}$ If the structure deviates from the ideal square lattice, various ways to form a spin-gap become possible. ${ }^{9)} \mathrm{Un}$ ambiguous determination of the structure is crucial for good understanding of a frustrated quantum spin system.

In this paper, we report magnetization, nuclear magnetic resonance (NMR), nuclear quadrupole resonance (NQR), and transmission electron microscopy (TEM) studies on $(\mathrm{CuCl}) \mathrm{LaNb}_{2} \mathrm{O}_{7}$. Our results indicate that 


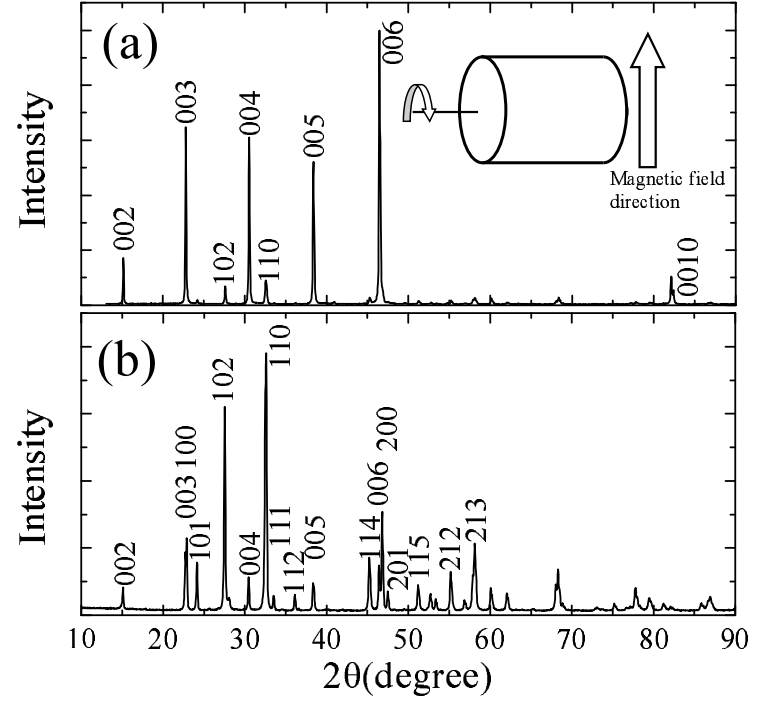

Fig. 2. X-ray diffraction patterns of the (a) aligned and (b) unaligned powder samples. The inset illustrates the method for preparing the aligned sample.

none of the $\mathrm{Cu}, \mathrm{Cl}$, and La sites has the $C_{4}$-symmetry, incompatible with the reported tetragonal structure. Thus the $J_{1}-J_{2}$ model for the simple square lattice has to be modified. We propose two-dimensional dimer models which are consistent with our NMR, NQR, and TEM results. $\mathrm{Cu}$ - and $\mathrm{Nb}-\mathrm{NMR}$ signals disappear above the critical field $B_{c 1} \simeq 10.3 \mathrm{~T}$ at low temperatures, indicating a field-induced magnetic phase transition at $B_{c 1}$.

\section{Experiment}

The powder sample of $(\mathrm{CuCl}) \mathrm{LaNb}_{2} \mathrm{O}_{7}$ was synthesized by the ion-exchange reaction of $\mathrm{RbLaNb}_{2} \mathrm{O}_{7}$ with $\mathrm{CuCl}_{2} .{ }^{4,7)}$ The magnetically aligned sample was prepared by rotating a cylindrical tube filled with the $(\mathrm{CuCl}) \mathrm{LaNb}_{2} \mathrm{O}_{7}$ powder and epoxy (STYCAST1266) in a magnetic field $B=7 \mathrm{~T}$. The magnetic field was applied perpendicular to the rotating axis as shown in the inset of Fig. 2. In this configuration, the magnetic hard axis tends to align along the rotating axis. The X-ray diffraction pattern for the aligned sample was obtained with a MacScience M03X diffractometer using a monochromatic $\mathrm{Cu}-K \alpha$ radiation as shown in Fig. 2 (a). Here the aligned axis was kept perpendicular to the scattering wave vector. Compared with the diffraction pattern for the unaligned powder shown in Fig. 2 (b), the peaks corresponding to $(00 n)$ planes are strongly enhanced in the aligned sample, where $n$ is integer. This indicates that the $c$-axis is the magnetic hard axis. The rocking curve for these peaks with the half width of about $1.5^{\circ}$ shows successful alignment. However, other peaks such as (102) or (110) are also observed in the aligned sample, indicating that a certain fraction of the sample remains unaligned.

The magnetic susceptibility $\chi$ was measured for the unaligned powder sample with a SQUID magnetometer (Quantum Design, MPMS). NQR measurements in zero magnetic field have been performed for the ${ }^{63} \mathrm{Cu}$ and ${ }^{35} \mathrm{Cl}$ nuclei in the unaligned powder sample. Nuclear

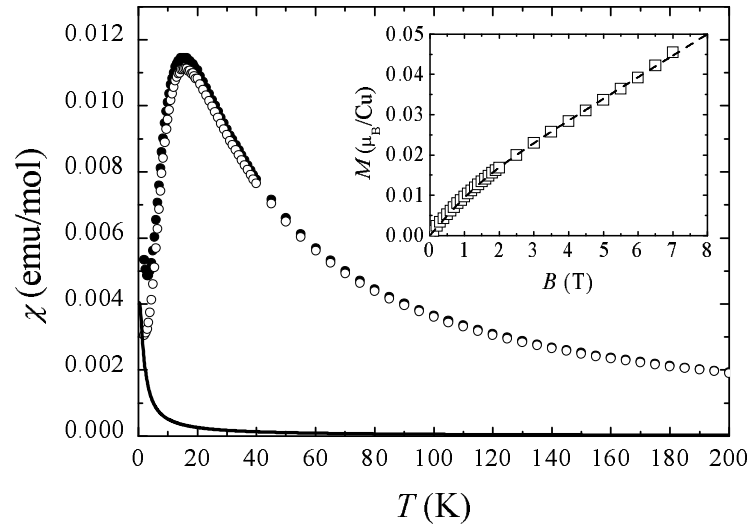

Fig. 3. Temperature dependence of $\chi$ measured for the unaligned powder sample at $1 \mathrm{~T}$. The solid circles represent the raw data. The open circles represent $\chi$ after subtracting the impurity contribution shown by the solid line. The inset shows the magnetic field $(B)$ dependence of the magnetization at $2 \mathrm{~K}$. The dashed line is the fit to the form $M=N g S \mu_{\mathrm{B}} B_{S}(x)+A B$.

spin-lattice relaxation rates were measured by the inversion recovery method. NMR measurements in magnetic fields up to $11.7 \mathrm{~T}$ have been performed on the aligned sample with the magnetic field applied along the $c$-axis for ${ }^{63,65} \mathrm{Cu},{ }^{35,37} \mathrm{Cl},{ }^{93} \mathrm{Nb}$, and ${ }^{139} \mathrm{La}$ nuclei. The TEM experiments were carried out at room temperature using a JEM2010F system with an operating voltage of $200 \mathrm{kV}$. The specimen was finely ground in methanol and then placed on a $\mathrm{Cu}$ microgrid mesh for TEM observation.

\section{Results and Analysis}

We first analyze the temperature dependence of $\chi$ in section 3.1. In section 3.2, we show the NQR results, which demonstrate that both $\mathrm{Cu}$ and $\mathrm{Cl}$ atoms occupy a unique site and there is no apparent disorder. The electric field gradient tensors at the $\mathrm{Cu}, \mathrm{Cl}$, and La sites are investigated from the field swept NMR spectra in section 3.3. These results indicate deviation of the crystal structure from the reported one with a simple square lattice. In section 3.4, the temperature dependences of the magnetic hyperfine shifts at the $\mathrm{Cu}$ and $\mathrm{Cl}$ sites are determined. Then the value of spin-gap is estimated. The hyperfine coupling constants are also determined at the $\mathrm{Cu}, \mathrm{Cl}$, and $\mathrm{Nb}$ sites. In section 3.5, the high field behavior above $B_{c 1}$ is investigated at the $\mathrm{Cu}$ and $\mathrm{Nb}$ sites. Finally, we show the TEM result in order to provide additional information about the structure.

\subsection{Magnetic susceptibility measurements}

Figure 3 shows the temperature $(T)$ dependence of $\chi$ measured at the field of $1 \mathrm{~T}$. The peak at $16 \mathrm{~K}$ and rapid decrease at lower temperatures are quite consistent with the previous report, ${ }^{4)}$ indicating a singlet ground state. Below $4 \mathrm{~K}$, however, $\chi$ increases with decreasing temperature probably due to magnetic impurities. In order to investigate the impurity contribution, we also measured magnetization $M$ up to $7 \mathrm{~T}$. The inset of Fig. 3 shows the $B$-dependence of $M$ at $2 \mathrm{~K}$. The data can be fitted by the sum of a term proportional to the Brillouin function $B_{S}(x)$ and a $B$-linear compo- 

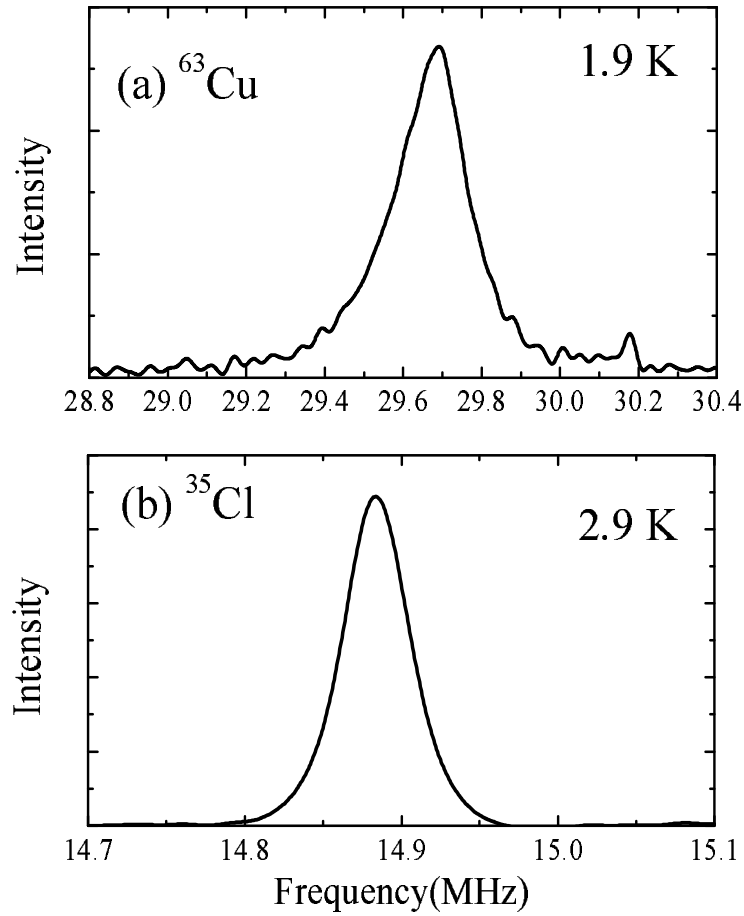

Fig. 4. NQR spectra (a) at the ${ }^{63} \mathrm{Cu}$ sites $(T=1.9 \mathrm{~K})$ and (b) at the ${ }^{35} \mathrm{Cl}$ sites $(T=2.9 \mathrm{~K})$.

nent $M=N g S B_{S}(x)+A B\left(x=g S \mu_{\mathrm{B}} B / k_{\mathrm{B}} T\right)$. A good agreement was obtained for the values $S=1 / 2, N=0.72$ $\% / \mathrm{Cu}$, and $N_{\mathrm{A}} \mu_{\mathrm{B}} A=3.0 \times 10^{-3} \mathrm{emu} / \mathrm{mol}\left(N_{\mathrm{A}}\right.$ is the Avogadro constant) as shown by the dashed line in the inset of Fig. 3. The first term leads to the free spin contribution to $\chi, N_{\mathrm{A}} N g S \mu_{\mathrm{B}} B_{S}(x) / B$, due to isolated magnetic impurities (the solid line in Fig. 3). By subtracting this term from the measured $\chi$, we obtained the corrected $\chi$ shown by the open circles. The corrected $\chi$ maintains a finite value $\left(3 \times 10^{-3} \mathrm{emu} / \mathrm{mol}\right)$ as $T$ goes to 0 . It is also detected in the magnetization curve as the $B$-linear component. This is much larger than the typical value of the Van Vleck susceptibility of $\mathrm{Cu}^{2+}\left(10^{-5} \sim 10^{-4}\right.$ $\mathrm{emu} / \mathrm{mol}$ ). The NMR shifts at all sites do not show such large residual values at low temperatures as we discuss later. Hence the large residual susceptibility at low $T$ is considered to be extrinsic, e.g. due to a secondary phase.

\subsection{NQR spectra and relaxation rates}

Figure 4 shows the NQR spectra at zero field for the ${ }^{63} \mathrm{Cu}$ and ${ }^{35} \mathrm{Cl}$ sites, both having the nuclear spin $I=$ $3 / 2$. A sharp single NQR line is observed for each nuclei. This shows that both $\mathrm{Cu}$ and $\mathrm{Cl}$ atoms occupy a unique site and there is no substantial disorder for the $\mathrm{Cu}$ and $\mathrm{Cl}$ positions. The NQR frequencies $\nu_{N Q R}$ are determined to be $29.68 \mathrm{MHz}$ for ${ }^{63} \mathrm{Cu}$ and $14.88 \mathrm{MHz}$ for ${ }^{35} \mathrm{Cl}$. The values of $\nu_{N Q R}$ and the spectrum shape show no temperature dependence from $10 \mathrm{~K}$ to $1.5 \mathrm{~K}$, indicating that the structure is unchanged at low temperatures.

Figure 5 shows the inverse temperature dependence of $1 / T_{1}$ at the ${ }^{63} \mathrm{Cu}$ and ${ }^{35} \mathrm{Cl}$ sites at zero field. At both sites, $1 / T_{1}$ is well fitted by an activation law $1 / T_{1} \propto$ $\exp \left(-E / k_{\mathrm{B}} T\right)$. The activation energy is obtained as $E / k_{\mathrm{B}}=23 \pm 2 \mathrm{~K}$ for $\mathrm{Cu}$ and $E / k_{\mathrm{B}}=21 \pm 1 \mathrm{~K}$ for

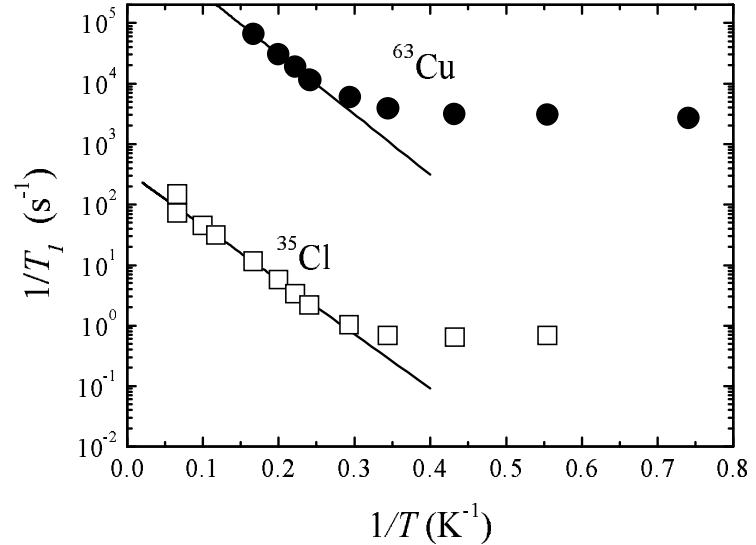

Fig. 5. Inverse temperature dependence of $1 / T_{1}$ at the ${ }^{63} \mathrm{Cu}$ and ${ }^{35} \mathrm{Cl}$ sites. The solid lines show the fit to an activation law $1 / T_{1} \propto$ $\exp \left(-E / k_{\mathrm{B}} T\right)$.

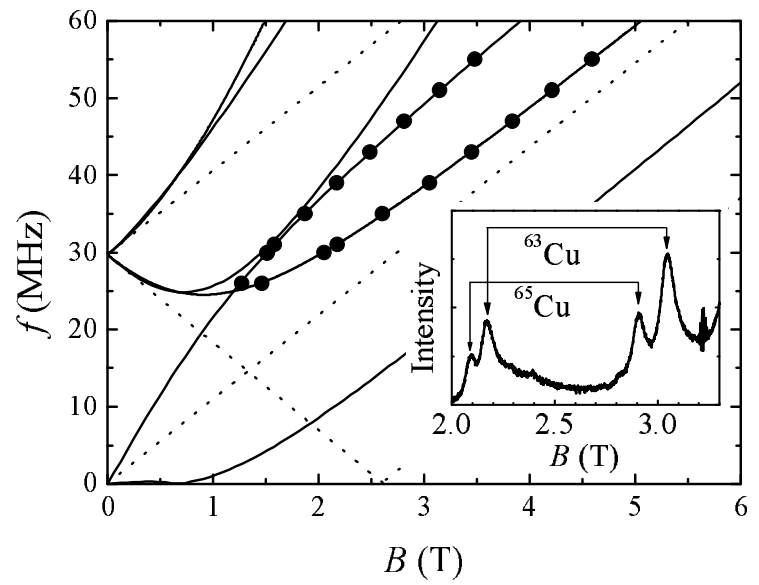

Fig. 6. Frequency-field diagram at $3 \mathrm{~K}$ at the ${ }^{63} \mathrm{Cu}$ sites in the applied magnetic fields $B$ parallel to the $c$-axis. The dotted lines represent the resonance modes for the case of axially symmetric EFG around the $c$-axis. The solid lines are the fit to the results obtained from the Hamiltonian (2). The inset shows the $\mathrm{Cu}-$ NMR spectrum at $43 \mathrm{MHz}$.

Cl. Below $4 \mathrm{~K}, 1 / T_{1}$ deviates from the activation law and become independent of temperature below $3 \mathrm{~K}$. The origin of the constant relaxation rate at low temperatures is likely to be impurities.

\subsection{Field swept NMR spectra and EFG tensors}

In the inset of Fig. 6, we show a typical field swept $\mathrm{Cu}$ NMR spectrum for the aligned sample with the magnetic field $B$ applied parallel to the $c$-axis. The spectrum has four sharp peaks assigned to the ${ }^{63} \mathrm{Cu}$ - and ${ }^{65} \mathrm{Cu}-\mathrm{NMR}$ lines from the aligned part of the sample. The spectrum also shows a broad tail spreading to higher field. This tail probably comes from the unaligned portion of the sample. The resonance fields are determined precisely from the peak positions. The frequency-field $(f-B)$ diagram can be constructed by plotting the resonance fields for various NMR frequencies between 26 and $55 \mathrm{MHz}$ as shown in Fig. 6.

The resonance frequency is generally determined from 


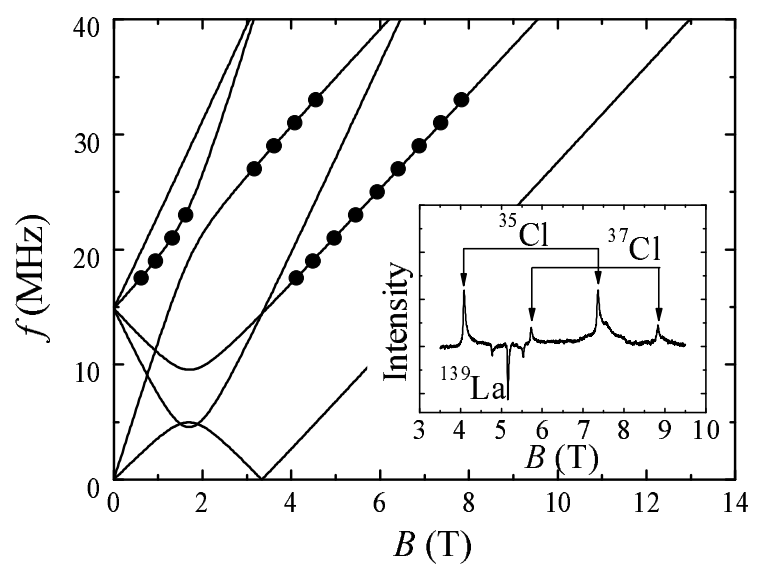

Fig. 7. Frequency-field diagram at $3 \mathrm{~K}$ at the ${ }^{35} \mathrm{Cl}$ sites in the applied magnetic fields $B$ parallel to the $c$-axis. The solid lines show the fit obtained from the Hamiltonian (2). The inset shows the $\mathrm{Cl}-\mathrm{NMR}$ spectrum at $31 \mathrm{MHz}$.

the following Hamiltonian including the magnetic hyperfine interaction and the electric quadrupole interaction, ${ }^{10)}$

$$
\begin{aligned}
H= & -h \gamma(\boldsymbol{B} \cdot \boldsymbol{I}+\boldsymbol{I} \cdot \hat{K} \cdot \boldsymbol{B}) \\
& +\frac{e Q}{6 I(2 I-1)} \sum_{\alpha, \beta} V_{\alpha \beta}\left[\frac{3}{2}\left(I_{\alpha} I_{\beta}+I_{\beta} I_{\alpha}\right)-\delta_{\alpha \beta} I^{2}\right],
\end{aligned}
$$

where $h$ is the Planck's constant, $Q$ is the nuclear quadrupole moment, $\gamma$ is the gyromagnetic ratio, $\hat{K}$ is the magnetic hyperfine shift tensor, $\delta_{\alpha \beta}$ is $0(\alpha \neq \beta)$ or 1 $(\alpha=\beta)$, and $V_{\alpha \beta}=\partial^{2} V / \partial \alpha \partial \beta(\alpha, \beta=x, y$, or $z)$ is the component of the electric field gradient (EFG) tensor. If the $\mathrm{Cu}$ sites have the $C_{4}$-symmetry around the $c$-axis in the tetragonal crystal structure, $V_{\alpha \beta}$ is axially symmetric, that is, $V_{\alpha \beta}=0$ for $\alpha \neq \beta$ and $V_{z z}=-2 V_{x x}$ $\left(V_{x x}=V_{y y}, z \| c\right)$. If the magnetic field is applied parallel to the symmetric $c$-axis, three resonance modes appear at the frequencies $\nu_{R}$ and $\left|\nu_{N Q R} \pm \nu_{R}\right|$, where $\nu_{R}=\left(1+K_{c c}\right) \gamma B$ and $\nu_{N Q R}=3 e Q V_{z z} / 2 I(2 I-1) h$, provided that all the components of $\hat{K}$ are much smaller than one. These results shown by the straight dotted lines in Fig. 6 are apparently in contradiction with the nonlinear behavior of the experimental data. This gives the direct evidence that the $\mathrm{Cu}$ sites do not have axial symmetry around the $c$-axis.

In the following, we assume that the $c$-axis is one of the principal axes of the EFG tensor at the $\mathrm{Cu}$ sites. This is the case, for example, when the $\mathrm{Cu}-\mathrm{Cl}$ planes have the mirror symmetry. Although this assumption may not be strictly valid in the real structure, it is not possible to determine $V_{\alpha \beta}$ from the available data without any assumption. The nuclear Hamiltonian (1) is rewritten for the magnetic field parallel to the $c$-axis $(z$-axis) as

$$
\begin{aligned}
H= & -h \gamma B(1+K) I_{z} \\
& +\frac{h}{3}\left(\nu_{x} I_{x}^{2}+\nu_{y} I_{y}^{2}+\nu_{z} I_{z}^{2}\right),
\end{aligned}
$$

where $K$ is the magnetic hyperfine shift along the $c$-axis

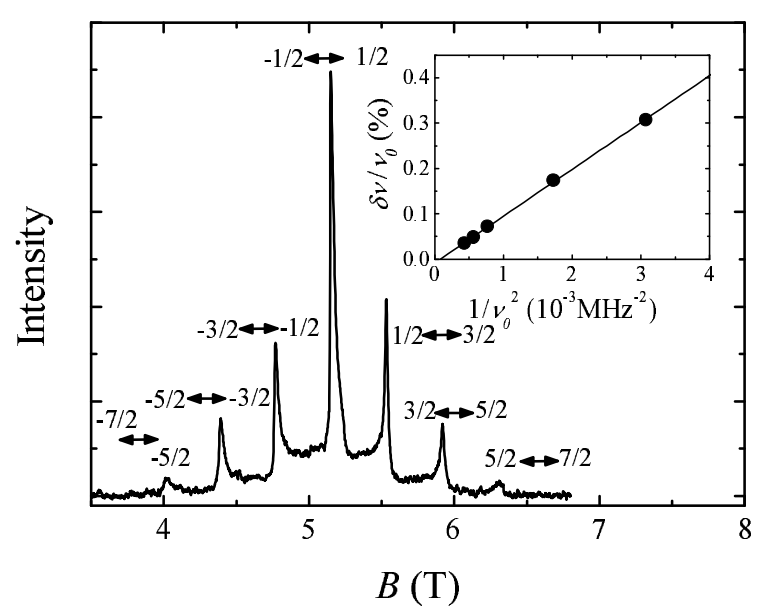

Fig. 8. Field swept NMR spectrum at the ${ }^{139}$ La sites at $6 \mathrm{~K}$ and $31 \mathrm{MHz}$ in the magnetic field parallel to the $c$-axis. The inset shows the $\delta \nu / \nu_{0}$ versus $1 / \nu_{0}^{2}$ plot at $115 \mathrm{~K}$.

and $\nu_{\alpha}=\{3 e Q / 2 I(2 I-1) h\} V_{\alpha \alpha} \cdot{ }^{10)}$ Although eq. (2) contains four parameters $\left(\nu_{z}, \nu_{x}, \nu_{y}\right.$, and $\left.K\right)$, the number of independent parameters can be reduced to two $\left(K\right.$ and $\left.\nu_{z}\right)$ from the relations $\nu_{x}+\nu_{y}+\nu_{z}=0$ and $\nu_{N Q R}=\sqrt{\nu_{z}^{2}+\left(\nu_{x}-\nu_{y}\right)^{2} / 3}$, where the NQR frequency $\nu_{N Q R}$ is determined to be $29.68 \mathrm{MHz}$ in section 3.1. Four eigenvalues of this Hamiltonian can be calculated analytically in the case of $I=3 / 2$, resulting in six resonance modes. As shown in Fig. 6, the experimental data are well fitted by the solid lines obtained from eq. (2) with $\nu_{z}=16.26 \pm 0.02 \mathrm{MHz}, \nu_{x}=13.37 \pm 0.02 \mathrm{MHz}, \nu_{y}=$ $-29.64 \pm 0.02 \mathrm{MHz}$, and $K=0.44 \pm 0.02 \%$.

Figure 7 shows the $f-B$ diagram at the ${ }^{35} \mathrm{Cl}$ sites obtained from the field swept NMR spectra of the aligned sample. A typical spectrum is shown in the inset of Fig. 7. The applied magnetic field $B$ was parallel to the aligned direction (c-axis). The spectrum also shows the ${ }^{139} \mathrm{La}-$ NMR lines. The negative intensity is due to improper rf-pulse conditions, which were optimized for $\mathrm{Cl}$ signal. The $f-B$ diagram can be fitted by solving the Hamiltonian (2) in the same way as has been done for the $\mathrm{Cu}$ sites. In Fig. 7, the solid lines show the results calculated for the parameter values $\nu_{z}=14.17 \pm 0.02 \mathrm{MHz}, \nu_{x}=$ $-3.13 \pm 0.02 \mathrm{MHz}, \nu_{y}=-11.04 \pm 0.02 \mathrm{MHz}$, and $K=$ $0.04 \pm 0.02 \%$.

Figure 8 shows the field swept ${ }^{139} \mathrm{La}$-NMR spectrum at $6 \mathrm{~K}$ and $31 \mathrm{MHz}$ in the magnetic field parallel to the $c$ axis. The seven peaks split by the quadrupole interaction for $I=7 / 2$ are clearly observed. Since the quadrupole interaction at the La sites is much smaller than that at the $\mathrm{Cu}$ and $\mathrm{Cl}$ sites, the second order perturbation theory is sufficient to extract the values of $\nu_{\alpha}$ and $K$. We assume the $c$-axis to be one of the principal axes for EFG. The resonance frequency between the states $|m\rangle$ and $|m-1\rangle$ is obtained from the Hamiltonian (2) as

$$
\begin{aligned}
\nu_{m \leftrightarrow m-1}= & \nu_{0}(1+K)-\left(m-\frac{1}{2}\right) \nu_{z} \\
& -\frac{\left(\nu_{x}-\nu_{y}\right)^{2}}{12 \nu_{0}}\left\{m(m-1)-\frac{19}{4}\right\}
\end{aligned}
$$




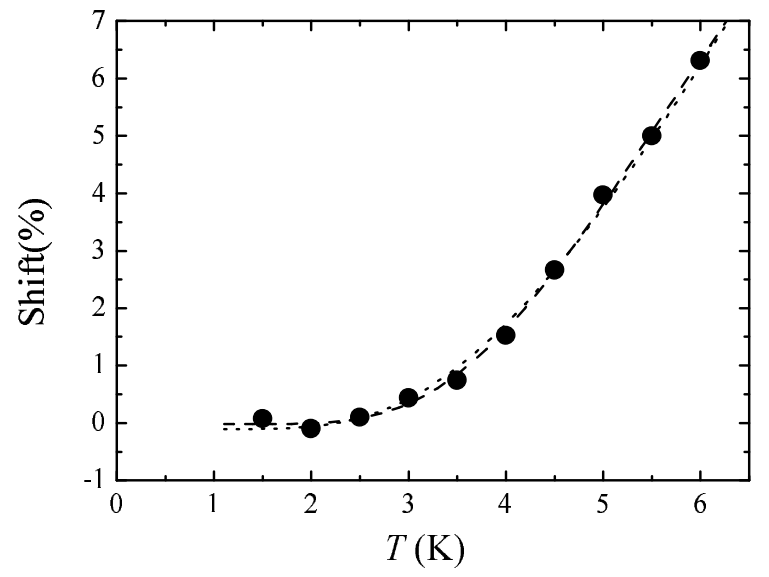

Fig. 9. Temperature dependence of $K$ at the ${ }^{63} \mathrm{Cu}$ sites for the magnetic field parallel to the $c$-axis. The dashed and dotted lines show the calculated results for the isolated dimer model and the two dimensional coupled dimer model, respectively.

where $\nu_{0}=\gamma B .{ }^{11)}$ If we define the frequency shift $\delta \nu_{m} \equiv$ $\nu_{m \leftrightarrow m-1}-\nu_{0}$, the frequency shift of the central line $\delta \nu_{1 / 2}$ obeys the relation,

$$
\frac{\delta \nu_{1 / 2}}{\nu_{0}}=K+\frac{5}{12} \frac{\left(\nu_{x}-\nu_{y}\right)^{2}}{\nu_{0}^{2}} .
$$

Therefore, $\delta \nu_{1 / 2} / \nu_{0}$ plotted against $1 / \nu_{0}^{2}$ should yield a straight line with the slope $(5 / 12)\left(\nu_{x}-\nu_{y}\right)^{2}$ and the intercept $K$ at $1 / \nu_{0}^{2}=0$. The inset of Fig. 8 shows the $\delta \nu / \nu_{0}$ versus $1 / \nu_{0}^{2}$ plot at $115 \mathrm{~K}$. We find that $K$ at the La sites is almost zero $\left(-8 \times 10^{-3} \pm 2 \times 10^{-3} \%\right)$ and $\left|\nu_{x}-\nu_{y}\right|=1.57 \mathrm{MHz}$. The value of $\nu_{z}$ is determined from the frequency shift for the satellite lines as $\nu_{z}=\left|\nu_{m \leftrightarrow m-1}-\nu_{-m+1 \leftrightarrow-m}\right| /(2 m-1)$. The values of $\nu_{z}, \nu_{x}$, and $\nu_{y}$ are determined to be $2.23 \pm 0.02,-0.33$ \pm 0.02 , and $-1.90 \pm 0.02 \mathrm{MHz}$, respectively.

The values of $\nu_{\alpha}$ determined by NMR are listed in Table I for the $\mathrm{Cu}, \mathrm{Cl}$, and La sites. It should be noted that the values in Table I are determined on the assumption that the $c$-axis is one of the principal axes of EFG. The EFG tensor is not axially symmetric around the $c$-axis for all sites. That is, none of these sites has the $C_{4}$-symmetry, which is incompatible with the reported tetragonal structure. Thus the $J_{1}-J_{2}$ model for the simple square lattice has to be modified for proper description of this system. We discuss modified models in section 4.3.

\subsection{Hyperfine shift and coupling constant}

By repeating the above analysis for different temperatures, we obtained the temperature dependence of $K$. Figure 9 shows the temperature dependence of $K$ at the $\mathrm{Cu}$ sites in the magnetic field parallel to the $c$-axis. The

Table I. Principal values of EFG tensors at the $\mathrm{Cu}, \mathrm{Cl}$, and $\mathrm{La}$ sites.

\begin{tabular}{|c|c|c|c|}
\hline & $\mathrm{Cu}$ & $\mathrm{Cl}$ & La \\
\hline$\nu_{z}(\mathrm{MHz})$ & $16.26 \pm 0.02$ & $14.17 \pm 0.02$ & $2.23 \pm 0.02$ \\
\hline$\nu_{x}(\mathrm{MHz})$ & $13.37 \pm 0.02$ & $-3.13 \pm 0.02$ & $-0.33 \pm 0.02$ \\
\hline$\nu_{y}(\mathrm{MHz})$ & $-29.64 \pm 0.02$ & $-11.04 \pm 0.02$ & $-1.90 \pm 0.02$ \\
\hline
\end{tabular}

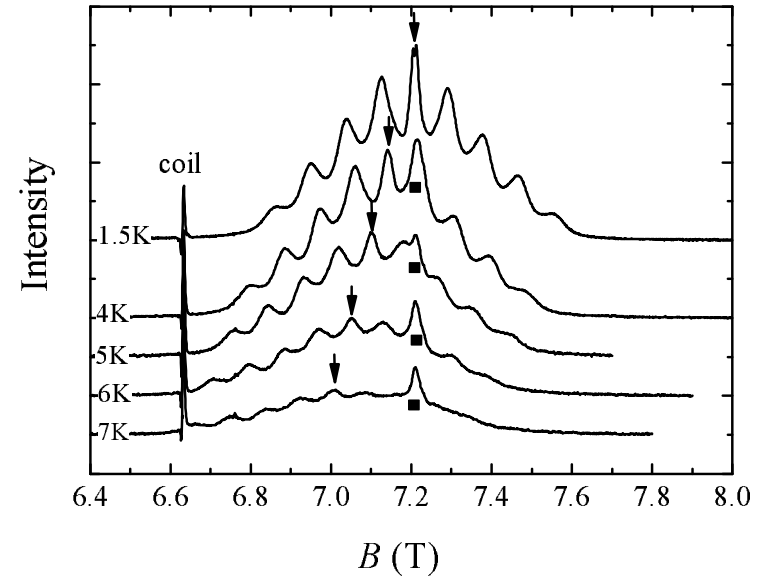

Fig. 10. Temperature dependence of the field swept spectrum at the $\mathrm{Nb}$ sites at $75 \mathrm{MHz}$ for the applied magnetic fields $B$ parallel to the $c$-axis.

shift consists of the contributions from the spin and Van Vleck susceptibility at the Cu sites, $K=K_{s}+K_{\mathrm{VV}}$. Each term is related to the spin and the Van Vleck susceptibility as $K_{s}=\left(A_{h f} / N_{\mathrm{A}} \mu_{\mathrm{B}}\right) \chi_{s}, K_{\mathrm{VV}}=\left(A_{\text {orb }} / N_{\mathrm{A}} \mu_{\mathrm{B}}\right) \chi_{\mathrm{VV}}$. The chemical shift is expected to be negligibly small compared with the spin and orbital shifts due to $d$-electrons of $\mathrm{Cu}^{2+}$. As shown in Fig. 9, $K$ decreases with decreasing $T$ and is extrapolated to zero at $T=0$. This means $K_{s}$ and $A_{h f}$ are positive. Since $K_{\mathrm{VV}}$ is always positive, we can conclude that $K_{\mathrm{VV}}$ is nearly zero and $K_{s}$ approaches zero at $T=0$. It should be noted that while $\chi$ contains impurity contribution, $K$ is free from impurities, and thus provides direct evidence for a spin-gap.

We estimate the spin-gap in two different models. The dashed line in Fig. 9 is the fit to the isolated dimer model

$$
K=K_{\mathrm{VV}}+\frac{C}{T}\left[1+\frac{1}{3} \exp \left(E^{\prime} / k_{\mathrm{B}} T\right)\right]^{-1},
$$

from which we obtained $E^{\prime} / k_{\mathrm{B}}=21.8 \mathrm{~K}$ and $K_{\mathrm{VV}}=$ $-0.02 \pm 0.02 \%$. Note that the shift was determined in the range of magnetic field from 1 to $5 \mathrm{~T}$. Considering the reduction of the gap by the average field $g \mu_{\mathrm{B}}\langle B\rangle / k_{\mathrm{B}} \simeq$ $4 \mathrm{~K}(\langle B\rangle=3 \mathrm{~T})$, the gap at zero field is estimated to be $26 \mathrm{~K}$. This value is rather close to the gap $(\sim 27 \mathrm{~K})$ estimated from the neutron inelastic scattering and the susceptibility measurements. ${ }^{4,5)}$ However, the isolated dimer model ignores the dispersion of the triplet excitations. In the opposite limit, where the triplets have large dispersion $\epsilon_{q}=E^{\prime \prime}+c q^{2}$, the spin susceptibility is determined by the excitation near the bottom of the dispersion $K_{s} \propto(1 / T) \int D(\epsilon) n(\epsilon) d \epsilon \propto \exp \left(-E^{\prime \prime} / K_{\mathrm{B}} T\right)$, where $D(\epsilon)$ is the density of state, which is constant for $\epsilon \geq E^{\prime \prime}$ in two dimension, and $n(\epsilon)$ is the Bose factor. The dotted line in Fig. 9 shows the fit to this model with $E^{\prime \prime} / k_{\mathrm{B}}=15 \mathrm{~K}$. Therefore, the gap is estimated to be 19 $\mathrm{K}$ at zero field. The gap values obtained in this study are compared with the reported results in section 4.1.

Figure 10 shows the temperature dependence of the field swept NMR spectrum at the $\mathrm{Nb}$ sites. The nine peaks for $I=9 / 2$ is observed at $1.5 \mathrm{~K}$ and the central line is indicated by the arrow. The central and the eight 


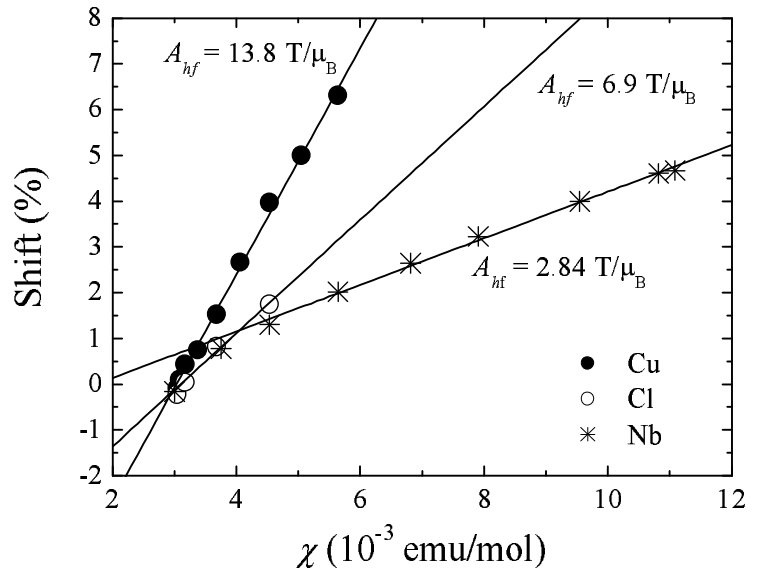

Fig. 11. $K-\chi$ plot for the $\mathrm{Cu}, \mathrm{Cl}$, and $\mathrm{Nb}$ sites.

satellite lines shift to lower fields with increasing temperature, indicating sizable hyperfine coupling at the $\mathrm{Nk}$ sites to spin magnetization of $\mathrm{Cu}$ ions. In addition, we observed another peak marked by the solid square in Fig. 10. This peak does not shift with temperature, indicating no hyperfine coupling to the $\mathrm{Cu}$ spins. In fact: $1 / T_{1}$ for this peak is ten times smaller than that for the intrinsic signal at low temperatures. Therefore, we conclude that this signal comes from extrinsic nonmagnetic phases. Imperfection of the ion-exchange reaction is a possible origin of such nonmagnetic impurely phases.

Figure 11 shows the $K-\chi$ plot for various sites obtained for the temperature range $1.5 \sim 20 \mathrm{~K}$. Here, a Curic term due to magnetic impurities was removed from the $\chi$ data as described in section 3.1. The hyperfine coupling constants $A_{h f}$ for $B \| c$ are determined to be $13.8 \pm 0.3$. $6.9 \pm 0.5$, and $2.84 \pm 0.08 \mathrm{~T} / \mu_{\mathrm{B}}$ for the $\mathrm{Cu}, \mathrm{Cl}$, and $\mathrm{Nb}$ sites, respectively, (Table II). Although the NMR shifts at all sites approach near zero as $T \rightarrow 0, \chi$ maintains a finite value $\left(3 \times 10^{-3} \mathrm{emu} / \mathrm{mol}\right)$ as mentioned in section 3.1. Note that the impurity term from free spins has been already subtracted. The residual $\chi$ at low temperatures, which corresponds to the $B$-linear magnetization in the inset of Fig. 3, does not couple to any nuclei observed by NMR. Hence we conclude that it has as extrinsic origin.

\subsection{High magnetic field region}

Figure 12 shows the field swept NMR spectrum at $1.5 \mathrm{~K}$ for the $\mathrm{Cu}$ sites at different frequencies in the higher magnetic field region above $B_{c 1} \simeq 10.3 \mathrm{~T}$. The very sharp peaks are due to the NMR-coil made from $\mathrm{Cu}$. The ${ }^{63,65} \mathrm{Cu}-\mathrm{NMR}$ lines from the aligned sample are indicated by the arrows. Their intensity decreases with increasing $B$ and the peaks from the aligned sample disappear slightly below $B_{c 1}=10.3 \mathrm{~T}$. This is because the spin-spin relaxation time $T_{2}$ is getting shorter when the field approaches $B_{c 1}$. There remains a broad signal near

Table II. Hyperfine coupling constants for $B \| c$.

\begin{tabular}{cccc}
\hline & $\mathrm{Cu}$ & $\mathrm{Cl}$ & $\mathrm{Nb}$ \\
\cline { 2 - 4 }$A_{h f}\left(\mathrm{~T} / \mu_{\mathrm{B}}\right)$ & $13.8 \pm 0.3$ & $6.9 \pm 0.5$ & $2.84 \pm 0.08$ \\
\hline
\end{tabular}

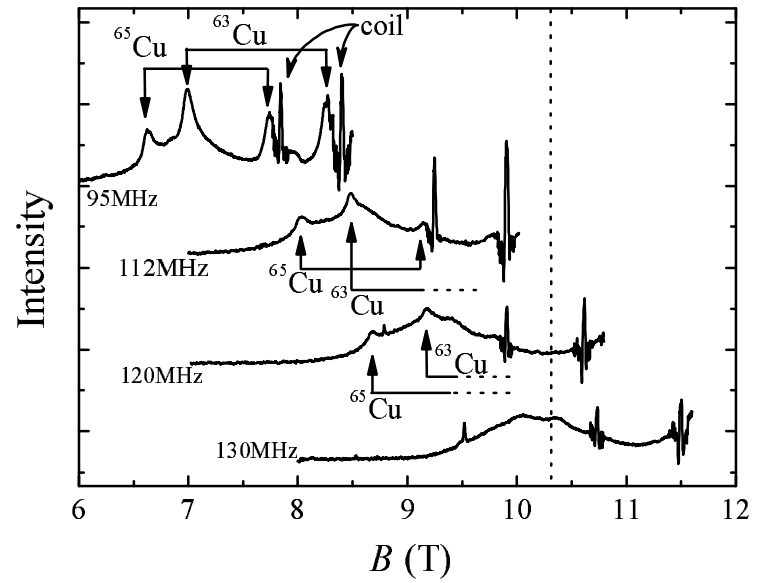

Fig. 12. Frequency dependence of the field swept NMR spectrum at the $\mathrm{Cu}$ sites at $1.5 \mathrm{~K}$ in the applied magnetic fields $B$ parallel to the $c$-axis. The dotted lines show the critical field $B_{c 1}$.

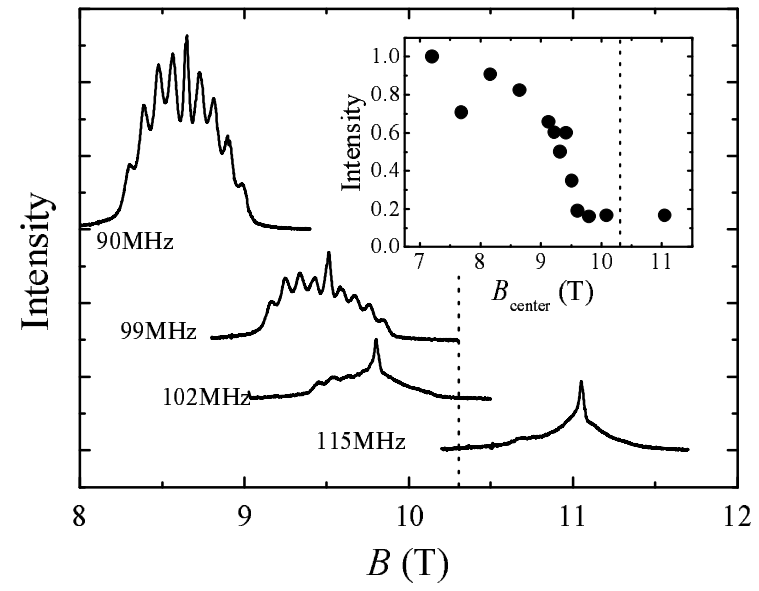

Fig. 13. Frequency dependence of the field swept NMR spectrum at the $\mathrm{Nb}$ sites at $1.5 \mathrm{~K}$ in the applied magnetic fields $B$ parallel to the $c$-axis. The inset shows the $B_{c e n t e r}$-dependence of the integrate intensity. The dotted lines show the critical field $B_{c 1}$.

$B_{c 1}$, observed in the spectrum at $130 \mathrm{MHz}$. Since this signal has much longer $T_{2}$, it is probably due to some impurity phases.

Figure 13 shows the frequency dependence of the field swept spectrum at the $\mathrm{Nb}$ sites. The quadruple split peaks for ${ }^{93} \mathrm{Nb}(I=9 / 2)$ are observed at $90 \mathrm{MHz}$. The intensity of the split peaks decreases with increasing $B$ and the signal disappears slightly below $B_{c 1}$ in a similar way as has been observed for the $\mathrm{Cu}$ signals. Above $B_{c 1}$, the spectrum with one central peak and broadened quadrupole satellites is observed $(115 \mathrm{MHz})$. This signal probably has the same origin as the unshifted signal observed at $75 \mathrm{MHz}$ at high temperatures (Fig. 10). In the inset of Fig. 13, the integrated intensity of the whole spectrum is plotted against the field of the central line $\left(B_{\text {center }}\right)$. The intensity normalized by the value at $7.2 \mathrm{~T}$ decreases with increasing $B_{\text {center }}$ and vanishes at $9.5 \mathrm{~T}$, slightly below $B_{c 1}$, except for the contribution from impurity phases, indicating that a field-induced magnetic phase transition occurs around $B_{c 1}$. The field-induced 

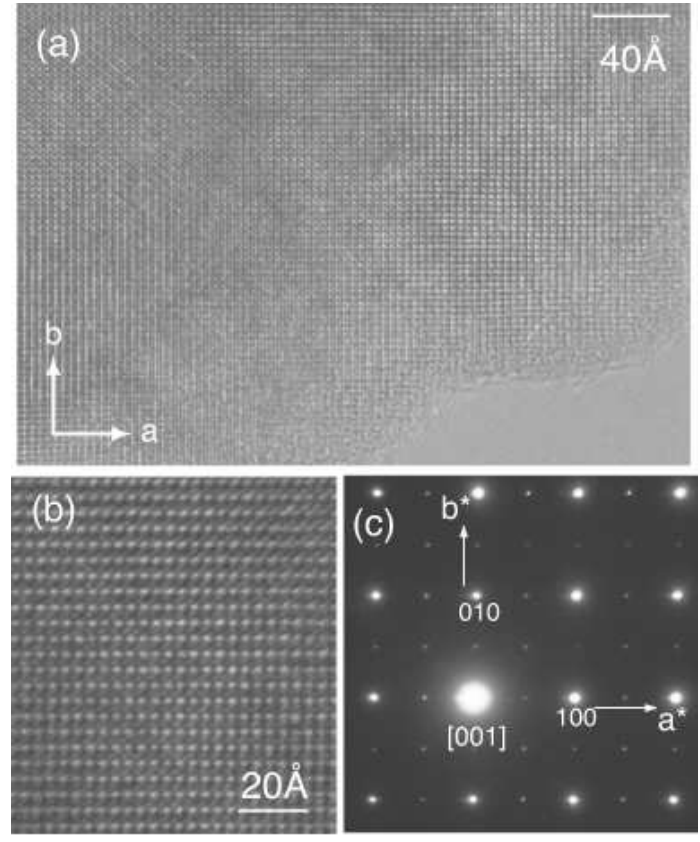

Fig. 14. (a) High-resolution TEM image of $(\mathrm{CuCl}) \mathrm{LaNb}_{2} \mathrm{O}_{7}$ at room temperature along the [001]-zone axis and (b) its enlarged view. (c) Corresponding electron diffraction pattern, where indices are given based on the tetragonal unit cell of the $P 4 / \mathrm{mmm}$ structure. ${ }^{7)}$

transition is also indicated by the recent specific heat measurements in magnetic fields. ${ }^{12)}$

\subsection{TEM result}

In order to obtain further information about the crystal structure, we performed TEM measurements at room temperature. Shown in Fig. 14 (a) and (b) are the [001] zone lattice images that demonstrate contrasts with a special period of about $4 \AA$ along $\langle 100\rangle$, consistent with the lattice parameter. However, the corresponding electron diffraction (Fig. 14 (c)) has revealed weak reflections such as $(1 / 200)$ or $(01 / 20)$, in addition to strong fundamental reflections relevant to the reported crystal structure. The weak reflections are commensurate and indicate the doubling of the lattice period of both $a$ - and $b$ axes, i.e., the size of the unit cell is given by $2 a \times 2 b$. Such a superstructure has not been detected even by high $q$ resolution synchrotron X-ray diffraction, ${ }^{13)}$ presumably due to tiny modulation of the atoms and/or small domain size. Note that the superstructure reflection at $(1 / 21 / 2$ 0 ) was observed in $(\mathrm{FeCl}) \mathrm{LaNb}_{2} \mathrm{O}_{7} \cdot{ }^{13)}$

\section{Discussion}

\subsection{Spin-gap}

The gap value in $(\mathrm{CuCl}) \mathrm{LaNb}_{2} \mathrm{O}_{7}$ is still controversial, while it has been investigated by several experimental methods. The most direct method is the inelastic neutron scattering, which shows the magnetic excitation with the gap energy of $2.3 \mathrm{meV}(26.7 \mathrm{~K}) .{ }^{4)}$ Although it is possible that the limited energy resolution masks fine structure of the neutron spectrum and the true gap may be somewhat smaller, the large discrepancy between this value and the gap derived from the critical magnetic field, $g \mu_{\mathrm{B}} B_{c 1} / k_{\mathrm{B}}$ (a)
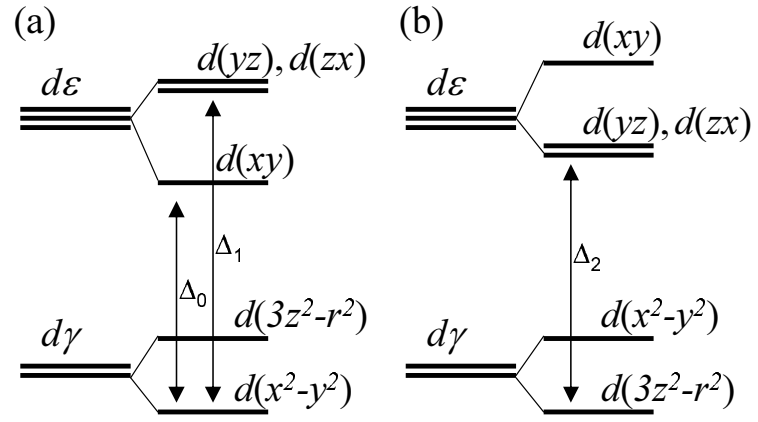

Fig. 15. Orbital energy levels for $\mathrm{Cu}^{2+}$ in a tetragonal crystal field. The ground state wave function is $d\left(x^{2}-y^{2}\right)$ in (a) or $d\left(3 z^{2}-r^{2}\right)$ in $(\mathrm{b})$.

$=15 \mathrm{~K}$, cannot be explained by such an effect.

From the susceptibility measurements, the gap is estimated to be $27 \mathrm{~K}$ by using the isolated dimer model. ${ }^{4)}$ The magnetic hyperfine shift determined by NMR is free from impurity contribution and provides more reliable spin susceptibility. Using the isolated dimer model, the gap value is determined to be $26 \mathrm{~K}$, consistent with the susceptibility results. We have also examined a twodimensional coupled dimer model with a large width of the triplet dispersion and obtained the gap value of $19 \mathrm{~K}$. Since these two models correspond to the opposite limits of zero and infinite width of the dispersion, the values 26 $\mathrm{K}$ and $19 \mathrm{~K}$ set the upper and the lower bounds for the true gap. From the NQR relaxation rate $1 / T_{1}$ at the $\mathrm{Cu}$ and $\mathrm{Cl}$ sites, the gap value is determined to be $23 \pm 2$ and $21 \pm 1 \mathrm{~K}$, respectively, consistent with the above range. This range is still significantly larger than $g \mu_{\mathrm{B}} B_{c 1} / k_{\mathrm{B}}(=$ $15 \mathrm{~K})$.

To reconcile the discrepancy between the gap estimated at low magnetic fields and $B_{c 1}$, Kageyama et al. proposed formation of two-triplet bound states with $S$ $=2{ }^{5)}$ If two-triplet bound states are stabilized due to frustration, the gap can close before $B$ reaches $\Delta / g \mu_{\mathrm{B}}$ and a nematic order may be realized above $B_{c 1} \cdot{ }^{14)}$ The $\mathrm{Cu}$ - and Nb-NMR signals were undetectable above $B_{c 1}$ at $1.5 \mathrm{~K}$ due to divergence of $1 / T_{2}$ as mentioned before (Figs. 12 and 13). To investigate the magnetic structure above $B_{c 1}$, we need measurements at higher fields and lower temperatures.

\section{2 d-orbital}

The experimental determination of $A_{h f}$ and $K_{\mathrm{VV}}$ at the $\mathrm{Cu}$ sites provides information about the $\mathrm{Cu} d$-orbital. In this section, we compare the experimental results of $A_{h f}$ and $K_{\mathrm{VV}}$ with the calculated values. Figure 15 shows the orbital energy levels for $\mathrm{Cu}^{2+}$ in tetragonal crystal fields. The $\mathrm{Cu}^{2+}$ ions have one $d$-hole with either $d\left(x^{2}-\right.$ $\left.y^{2}\right)$ or $d\left(3 z^{2}-r^{2}\right)$ wave function. The hyperfine coupling constants $A_{\|}^{h f}$ (for $B \| z$ ) and $A_{\perp}^{h f}($ for $B \perp z$ ) are 
expressed as

$$
\begin{aligned}
& A_{\|}^{h f}=\frac{\mu_{0} \mu_{\mathrm{B}}}{4 \pi}\left\langle r^{-3}\right\rangle\left(-\kappa-\frac{4}{7}+\frac{6}{7} \frac{\lambda}{\Delta_{1}}-\frac{8 \lambda}{\Delta_{0}}\right) \\
& A_{\perp}^{h f}=\frac{\mu_{0} \mu_{\mathrm{B}}}{4 \pi}\left\langle r^{-3}\right\rangle\left(-\kappa+\frac{2}{7}+\frac{11}{7} \frac{\lambda}{\Delta_{1}}\right) \text { for } d\left(x^{2}-y^{2}\right) \\
& A_{\|}^{h f}=\frac{\mu_{0} \mu_{\mathrm{B}}}{4 \pi}\left\langle r^{-3}\right\rangle\left(-\kappa+\frac{4}{7}+\frac{6}{7} \frac{\lambda}{\Delta_{2}}\right) \\
& A_{\perp}^{h f}=\frac{\mu_{0} \mu_{\mathrm{B}}}{4 \pi}\left\langle r^{-3}\right\rangle\left(-\kappa-\frac{2}{7}-\frac{45}{7} \frac{\lambda}{\Delta_{2}}\right) \text { for } d\left(3 z^{2}-r^{2}\right),
\end{aligned}
$$

where $\mu_{0}$ is the permeability of vacuum, $\kappa$ is the core polarization coefficient, $\lambda$ is the spin-orbit coupling parameter, and $\Delta_{i}(i=0,1,2)$ is the energy between the ground state and the excited sate shown in Fig. 15. ${ }^{15,16)}$ The values of $\kappa, \lambda$, and $\left\langle r^{-3}\right\rangle$ for $\mathrm{Cu}^{2+}$ ions are common to all insulating materials within $10-20 \%$, typically given as $\kappa=0.28, \lambda=-8.8 \times 10^{-2} \mathrm{eV}$, and $\left\langle r^{-3}\right\rangle=4.26 \times 10^{31}$ $\mathrm{m}^{-3}$.15,16) $\Delta_{i}$ depends on materials but should be of the order of a few eV. We take $\Delta_{i}=2.0 \mathrm{eV}{ }^{15,16)}$ By using these values, $A_{\|}^{h f}$ and $A_{\perp}^{h f}$ are estimated to be -24.1 and $2.71 \mathrm{~T} / \mu_{\mathrm{B}}$ for $d\left(x^{2}-y^{2}\right)$ and 10.2 and $-12.2 \mathrm{~T} / \mu_{\mathrm{B}}$ for $d\left(3 z^{2}-r^{2}\right)$, respectively. Compared with the experimental values, $A_{h f}\left(=13.8 \mathrm{~T} / \mu_{\mathrm{B}}\right), A_{\|}^{h f}$ for $d\left(x^{2}-y^{2}\right)$ and $A_{\perp}^{h f}$ for $d\left(3 z^{2}-r^{2}\right)$ can be excluded because they lead to large negative shifts. Note that the magnetic field is parallel to the $c$-axis in the experiment, and we consider the cases where the $z$-axis of the orbital is either parallel or perpendicular to the $c$-axis. Among the other two cases, $A_{\|}^{h f}$ for $d\left(3 z^{2}-r^{2}\right)$ is by far the favorable.

The vanishing Van Vleck susceptibility $K_{\mathrm{VV}} \simeq 0$ for $B \| c$ also supports the $d\left(3 z^{2}-r^{2}\right)$ orbital. For $B \| z$, the Van Vleck susceptibility $\chi_{\mathrm{VV}}(\mathrm{emu} / \mathrm{mol})$ is calculated as

$$
\chi_{\mathrm{VV}}=N_{\mathrm{A}} \mu_{\mathrm{B}}^{2} \sum_{n \neq 0} \frac{\left|\left\langle\psi_{n}\left|L_{z}\right| \psi_{0}\right\rangle\right|^{2}}{\epsilon_{n}-\epsilon_{0}},
$$

where $\psi_{0}$ is the grand state with the energy $\epsilon_{0}$ and $\psi_{n}$ is the excited state with the energy $\epsilon_{n}$. For the $d\left(3 z^{2}-r^{2}\right)$ orbital, $\chi_{\mathrm{VV}}$ is zero. On the other hand, $\chi_{\mathrm{VV}}$ for $d\left(z^{2}-x^{2}\right)$ is calculated to be $N_{\mathrm{A}} \mu_{\mathrm{B}}^{2} / \Delta_{1} \simeq 1.6 \times 10^{-5}$ $\mathrm{emu} / \mathrm{mol}$, leading to $K_{\mathrm{VV}}=2\left\langle r^{-3}\right\rangle \chi_{\mathrm{VV}} / N_{\mathrm{A}}=0.23 \%$. This is clearly incompatible with the experimental observation. Therefore, we conclude that the $\mathrm{Cu}$ spins are on the $d$-orbital consisting mainly of $d\left(3 z^{2}-r^{2}\right)$. This is natural because the $\mathrm{Cu}-\mathrm{O}$ bonding $(1.84 \AA)$ is much shorter than the $\mathrm{Cu}-\mathrm{Cl}$ bonding in the $a b$ plane (3.14 or $2.40 \AA) .{ }^{8)}$ Such structure and the difference of the ionic valence $\left(\mathrm{O}^{2-}\right.$ vs. $\left.\mathrm{Cl}^{-}\right)$make the $d\left(3 z^{2}-r^{2}\right)$ orbital favorable for the $d$-hole. It should be noted that broken axial symmetry revealed by our NMR data should allow slight mixing of other components such as $d\left(x^{2}-y^{2}\right)$. The large hyperfine field at the $\mathrm{Nb}$ sites can then be understood as due to the transferred hyperfine interaction through the $d\left(3 z^{2}-r^{2}\right)$ orbital. We suspect that the interplane exchange interaction through the path $\mathrm{Cu}-\mathrm{O}$ $\mathrm{Nb}-\mathrm{O}-\mathrm{Nb}-\mathrm{O}-\mathrm{Cu}$ and the nearest-neighbor exchange interaction through the path $\mathrm{Cu}-\mathrm{O}-\mathrm{O}-\mathrm{O}-\mathrm{Cu}$ may not be completely negligible.

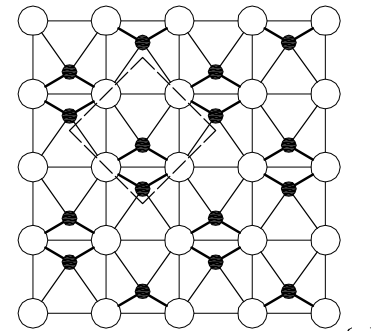

(a)
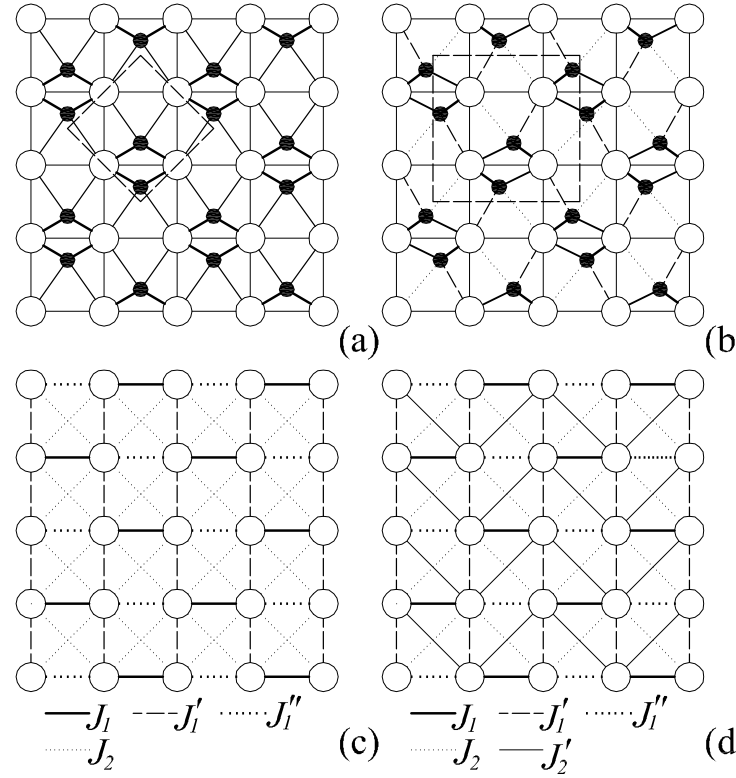

(d)

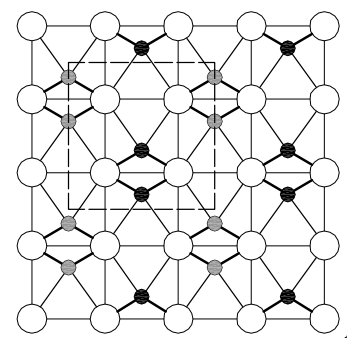

(e)

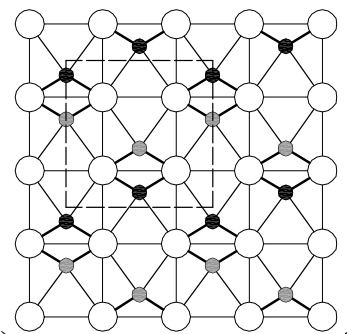

Fig. 16. Possible structures for the $\mathrm{CuCl}$ plane. The open and solid circles represent the $\mathrm{Cu}$ and $\mathrm{Cl}$ ions, respectively. The dashed lines in (a), (b), (e), and (f) indicate the unit cell for the superstructure. In the structure (e) and (f), the solid and gray spheres represent the $\mathrm{Cl}$ atoms above and below the $\mathrm{Cu}$ plane, respectively.

\subsection{Possible model}

Precise structural information of the $\mathrm{CuCl}$ plane is crucial to understand the origin of the spin-gap in this compound. Our NQR results show that both $\mathrm{Cu}$ and $\mathrm{Cl}$ have only one site and there is no apparent disorder. This means that periodic superstructure should be realized, even though the domain size of the superstructure may be very small. Although Caruntu et al. proposed disorder in the $\mathrm{Cl}$ position based on their neutron diffraction experiments, ${ }^{8)}$ their results are not necessarily incompatible with the NQR results because possibility of a superstructure was not considered in the analysis of the neutron data. In addition to the NQR results, the EFG tensor does not have axial symmetry at the $\mathrm{Cu}, \mathrm{Cl}$, and La sites and the TEM result shows that the unit cell is doubled along both the $a$ - and the $b$-axes from the original square lattice. Therefore, $J_{1}-J_{2}$ model for the simple square lattice has to be modified. Whangbo and Dai proposed models of ring clusters with even number of spins based on the neutron results to account for the spin-gap. They lead, however, to several inequivalent $\mathrm{Cu}$ and $\mathrm{Cl}$ sites, inconsistent with our NQR results.

The rather large spin-gap suggests that the real structure has such a feature that allows natural formation of 
$\mathrm{Cu}$ dimers. Figures 16 (a), (b), (e), and (f) show examples of such structures, where we assume that $\mathrm{Cu}$ maintains the ideal position for the square lattice, $(1 / 2,1 / 2,1 / 2)$. The structure in Fig. 16 (a) is simplest and leads to a two-dimensional dimer system as shown in Fig. 16 (c). Here, dimers are formed by the dominant exchange path $J_{1}$, and connected with each other by $J_{1}^{\prime}, J_{1}^{\prime \prime}$, and $J_{2}$. If $J_{1}$ is antiferromagnetic, a spin-gap is naturally formed. This structure has only one $\mathrm{Cu}$ and $\mathrm{Cl}$ site. The neutron diffraction study suggests that $\mathrm{Cl}$ moves off the ideal $(0$, $0,1 / 2)$ position to one of the $(x, 0,1 / 2),(-x, 0,1 / 2),(0$, $x, 1 / 2)$, and $(0,-x, 1 / 2)$ positions in a random manner. ${ }^{8)}$ The structure in (a) is an ordered version of the structure proposed from the neutron experiments. ${ }^{8)}$ However, the unit vectors $\hat{a} \pm \hat{b}$ of the structure (a) leads to the superstructure reflection at $(1 / 21 / 20)$ but cannot account for the observed TEM reflections such as $(1 / 20$ $0)$ and $\left(\begin{array}{lll}0 & 1 / 2 & 0\end{array}\right)$. Therefore, $\mathrm{Cl}$ should take more general positions. We propose three models (b), (e), and (f), which are consistent with both the NQR and TEM results. In the structure (b), $\mathrm{Cl}$ moves off the ideal $(0,0$, $1 / 2)$ position to a position, $(x, y, 1 / 2),(-x, y, 1 / 2),(x$, $-y, 1 / 2)$, or $(-x,-y, 1 / 2)$, still maintaining only one site. The dominant exchange path $J_{1}$ makes the dimers, which are connected by other exchange paths $J_{1}^{\prime}, J_{1}^{\prime \prime}, J_{2}$, and $J_{2}^{\prime}$ as shown in Fig. $16(\mathrm{~d})$. In the structures (e) and (f), Cl moves off to $(x, 0,1 / 2+\delta),(x, 0,1 / 2-\delta),(-x, 0$, $1 / 2+\delta)$, or $(-x, 0,1 / 2-\delta)$. The solid and gray spheres represent the $\mathrm{Cl}$ atoms above and below the $\mathrm{Cu}$ plane, respectively. In both (e) and (f), the exchange paths are the same as the structure (a) that is shown in Fig. 16 (c). Both models (c) and (d) generate frustration.

A useful insight is obtained by comparing the magnetic ground state of $(\mathrm{CuCl}) \mathrm{LaNb}_{2} \mathrm{O}_{7}$ and $(\mathrm{CuBr}) \mathrm{LaNb}_{2} \mathrm{O}_{7}$, which shows a collinear antiferromagnetic order. $\left.{ }^{6}\right) \mathrm{We}$ have observed a large internal field of $16 \mathrm{~T}$ at the ${ }^{79,81} \mathrm{Br}$ sites in $(\mathrm{CuBr}) \mathrm{LaNb}_{2} \mathrm{O}_{7}$ by zero field NMR. The details will be published in a separate paper. If $J_{1}$ is antiferromagnetic in $(\mathrm{CuBr}) \mathrm{LaNb}_{2} \mathrm{O}_{7}$, the internal field at the $\mathrm{Br}$ sites should be canceled out for the structures (a), (e), and (f). Therefore, if the two materials have the same structure, (b) is the only possibility. However, the exchange interaction through $\mathrm{Cl}$ or $\mathrm{Br}$ is very sensitive to small structural variation and often changes from ferromagnetic to antiferromagnetic. ${ }^{17)}$ Therefore, $J_{1}$ for $(\mathrm{CuBr}) \mathrm{LaNb}_{2} \mathrm{O}_{7}$ may be ferromagnetic, even if $J_{1}$ for $(\mathrm{CuCl}) \mathrm{LaNb}_{2} \mathrm{O}_{7}$ is antiferromagnetic. If $J_{1}$ is ferromagnetic in the structures (a), (e), and (f), a large internal field of the ordered moment survives. Therefore, both models (c) and (d) are possible. It seems that these examples exhaust possible structures within the condition of the uniqueness of the $\mathrm{Cu}$ and $\mathrm{Cl}$ sites and the doubled periodicity along the $a$ - and $b$-axes, although we are not able to prove rigorously.

\section{Summary}

NMR, NQR, and TEM results of $(\mathrm{CuCl}) \mathrm{LaNb}_{2} \mathrm{O}_{7}$ have been reported. A sharp single NQR line observed at each $\mathrm{Cu}$ and $\mathrm{Cl}$ site indicates that both $\mathrm{Cu}$ and $\mathrm{Cl}$ atoms occupy a unique site. The electric field gradient tensor shows that there is no axial symmetry around the $c$ axis at the $\mathrm{Cu}, \mathrm{Cl}$, and La sites. These results indicate that the $J_{1}-J_{2}$ model for the simple square lattice has to be modified. We propose a two-dimensional dimer model for this compound. $\mathrm{Cu}$ - and $\mathrm{Nb}-\mathrm{NMR}$ signals disappear above the critical field $B_{c 1} \simeq 10 \mathrm{~T}$ at low temperatures, indicating a field-induced magnetic phase transition occurs at $B_{c 1}$.

\section{Acknowledgment}

We would like to thank T. Momoi, N. Shannon, H. Nojiri and K.-Y. Choi for valuable discussions. This work was supported by a Grant-in-Aid for Scientific Research (Nos. 18740202 and 17684018) and a Grant-in-Aid on Priority Areas ("Invention of Anomalous Quantum Materials" Nos. 16076204 and 16076210) from the Ministry of Education, Culture, Sports, Science and Technology of Japan.

1) G. Misguich and C. Lhuillier: in "Frustrated spin systems", H. T. Diep Ed., (WorldScientific, Singapore 2003), condmat/0310405.

2) N. Read and S. Sachdev: Phys. Rev. Lett. 62 (1989) 1694.

3) N. Shannon, B. Schmidt, K. Penc and P. Thalmeier: Eur. Phys. J. B 38 (2004) 599.

4) H. Kageyama, T. Kitano, N. Oba, M. Nishi, S. Nagai, K. Hirota, L. Viciu, J. B. Wiley, J. Yasuda, Y. Baba, Y. Ajiro and K. Yoshimura: J. Phys. Soc. Jpn. 74 (2005) 1702.

5) H. Kageyama, J. Yasuda, T. Kitano, K. Totsuka, Y. Narumi, M. Hagiwara, K. Kindo, Y. Baba, N. Oba, Y. Ajiro and K. Yoshimura: J. Phys. Soc. Jpn. 74 (2005) 3155.

6) N. Oba, H. Kageyama, T. Kitano, J. Yasuda, Y. Baba, M. Nishi, K. Hirota, Y. Narumi, M. Hagiwara, K. Kindo, T. Saito, Y. Ajiro and K. Yoshimura: J. Phys. Soc. Jpn. 75 (2006) 113601.

7) T. A. Kodenkandath, J. N. Lalena, W. L. Zhou, E. E. Carpenter, C. Sangregorio, A. U. Falster, W. B. Simmons, C. J. O'Connor and J. B. Wiley: J. Am. Chem. Soc. 121 (1999) 10743.

8) G. Caruntu, T. A. Kodenkandath and J. B. Wiley: Mater. Res. Bull. 37 (2002) 593.

9) M.-H. Whangbo and D. Dai: Inorg. Chem. 45 (2006) 6227.

10) C. P. Slichter: "Principles of Magnetic Resonance" (SpringerVerlag Berlin Heidelberg, 1990).

11) G. H. Stauss: J. Chem. Phys. 40 (1964) 1988.

12) A. Kitada, Z. Hiroi, Y. Tsujimoto, T. Kitano, H. Kageyama, Y. Ajiro and K. Yoshimura: J. Phys. Soc. Jpn. 76 (2007) 093706.

13) N. Oba, H. Kageyama, T. Saito, M. Azuma, W. Paulus, T. Kitano, Y. Ajiro and K. Yoshimura: J. Magn. Magn. Matter. 310 (2007) 1337.

14) N. Shannon, T. Momoi and P. Sindzingre: Phys. Rev. Lett. 96 (2006) 027213.

15) A. Abragam and B. Bleaney: "Electron Paramagnetic Resonance of Transition Ions" (Oxford Univ. Press, New York, 1980).

16) C. H. Pennington, D. J. Durand, C. P. Slichter, J. P. Rice, E. D. Bukowski and D. M. Ginsberg: Phys. Rev. B 39 (1989) 2902.

17) Y. Inagaki, A. Kobayashi, T. Asano, T. Sakon, H. Kitagawa, M. Motokawa and Y. Ajiro: J. Phys. Soc. Jpn. 74 (2005) 2683. 